\title{
ENVIRONMENTAL INFLUENCES ON THE STABILITY OF A PERMANENTLY INSTALLED LASER SCANNER
}

\author{
M. Kuschnerus ${ }^{1, *}$, D. Schröder ${ }^{2,3, *}$, R. Lindenbergh ${ }^{1}$ \\ ${ }^{1}$ Department of Geoscience and Remote Sensing, Delft University of Technology, Stevinweg 1, 2628CN Delft, The Netherlands \\ \{m.kuschnerus, r.c.lindenbergh\}@tudelft.nl \\ ${ }^{2}$ Department of Civil and Mining Engineering, DMT GmbH \& Co. KG, Essen, Germany \\ daniel.schroeder@dmt-group.com \\ ${ }^{3}$ Faculty of Geoscience, Geotechnology and Mining, University of Mining and Technology Freiberg, Germany
}

\section{Commission II, WG II/10}

KEY WORDS: Laser Scanning, LIDAR, Multi-temporal 3D Point Cloud Analysis, Permanent Laser Scanning, Georeferencing, Error Budget.

\begin{abstract}
:
The advancement of permanently measuring laser scanners has opened up a wide range of new applications, but also led to the need for more advanced approaches on error quantification and correction. Time-dependent and systematic error influences may only become visible in data of quasi-permanent measurements. During a scan experiment in February/March 2020 point clouds were acquired every thirty minutes with a Riegl VZ-2000 laser scanner, and various other sensors (inclination sensors, weather station and GNSS sensors) were used to survey the environment of the laser scanner and the study site. Using this measurement configuration, our aim is to identify apparent displacements in multi-temporal scans due to systematic error influences and to investigate data quality for assessment of geomorphic changes in coastal regions. We analyse scan data collected around two storm events around 09/02/2020 (Ciara) and around 22/02/2020 (Yulia) and derive the impact of heavy storms on the point cloud data through comparison with the collected auxiliary data. To investigate the systematic residuals on data acquired by permanent laser scanning, we extracted several stable flat surfaces from the point cloud data. From a plane fitted through the respective surfaces of each scan, we estimated the mean displacement of each plane with the respective root mean square errors. Inclination sensors, internal and external, recorded pitch and roll values during each scan. We derived a mean inclination per scan (in pitch and roll) and the standard deviation from the mean as a measure of the stability of the laser scanner during each scan. Evaluation of the data recorded by a weather station together with knowledge of the movement behaviour, allows to derive possible causes of displacements and/or noise and correction models. The results are compared to independent measurements from GNSS sensors for validation. For wind speeds of $10 \mathrm{~m} / \mathrm{s}$ and higher, movements of the scanner considerably increase the noise level in the point cloud data.
\end{abstract}

\section{INTRODUCTION}

Terrestrial laser scanning (TLS) is an established measurement technique that has evolved continuously over the last two decades. Increasing operating ranges, the ability of automatic processing on the scanner itself or new algorithms for georeferencing are just a few examples of the improving functionality of sensor techniques. Various research groups use permanently installed laser scanners to address a wide range of research topics, including i²MON (Schröder and Klonowski, 2019) and CoastScan (Vos et al., 2017), (Kuschnerus et al., 2021). Disadvantages of conventional deformation analysis from single points may be overcome, new challenges arise or others remain (Wunderlich et al., 2016). The quality of results depends on many factors comprising the measuring system (Schröder and Nowacki, 2021). The appearance of permanently measuring sensors has made influences visible that were previously not apparent in campaign-based measurements at individual selected measurement times. Time-dependent, systematic error influences appear in time-series of quasi-permanent measurements (Schröder and Nowacki, 2021), (Friedli et al., 2019), (Anders et al., 2019).

As part of CoastScan and $\mathrm{i}^{2} \mathrm{MON}$, a fieldwork campaign in

\footnotetext{
* These authors contributed equally. Corresponding author: m.kuschnerus@tudelft.nl
}

Noordwijk, The Netherlands, took place in February and March 2020. In the course of this research experiment, the data from permanent laser scanning (PLS) are described qualitatively and influencing variables are quantified. Using this measurement configuration, the aim is to separate apparent displacements in the multi-temporal scans due to systematic error influences on the georeferencing and the travel time of the propagating laser beam from the real dynamics on the beach and in the dunes.

However, when analyzing and utilizing parts of this data set and another data set (Vos et al., 2020a), collected on a similar beach in Kijkduin with the same instrumental set-up, several systematic error effects became apparent. Among them is a day and night pattern seemingly correlated with temperature (Anders et al., 2019), as well as radial stripes visible above flat surfaces in some scans (see Figure 1). This effect occurs, for example, on 09/02/2020 in the evening hours. Two scans (09:00 and 19:00) are compared in Figure 1 with a reference mesh. For the reference, the scan on 09/02/2020 at 0:00 was selected. This leads to the assumption that despite an assumed stable and unchanged instrumental setup, (Vos et al., 2020b), systematic differences between selected point clouds occur.

Systematic deviations can lead to misinterpretations and indicate deformations in the point clouds that are not present in reality. A systematic deviation can only be taken into account in the 


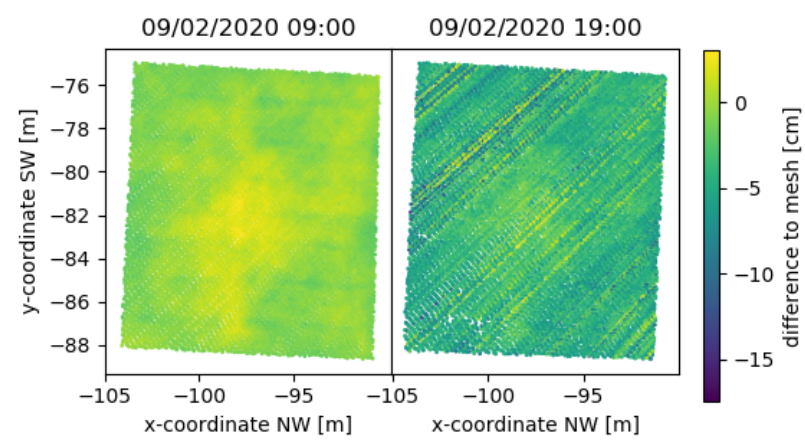

Figure 1. Residual plots of the helipad on 09/02/2020, at 09:00 (left) and at 19:00 (right). Colours represent distance to a reference mesh in $\mathrm{cm}$. On the right much larger differences in a striped pattern are visible.

measurement data if the cause is known. In the present case, the pattern occurs at certain points of time and is therefore clearly visible, which simplifies further investigations. Therefore, we focus on two research questions: What are causes of systematic error effects visible in the PLS data set? And how can these effects be separated and traced back to specific geometric and environmental conditions?

\section{RELATED WORK ON ERROR SOURCES}

Data from TLS is subject to various error sources in different scales. Due to the fixed setup and repeated scanning from the same position some error sources can be disregarded, whereas others appear more pronounced. There are absolute errors with respect to georeferencing and relative errors between the consecutive point clouds. The error sources influencing conventional TLS measurements are summarized by Soudarissanane et al. (Soudarissanane et al., 2011). Performance of TLS and the effects of the above mentioned error sources on point cloud data have been investigated by various research groups. They list the following main influences:

\section{Scanner mechanism}

\section{Atmospheric conditions and environment}

\section{Object properties}

\section{Scanning geometry.}

Errors from the scanner mechanism, as well as calibration methods are discussed in (Lichti, 2010). In addition the research group "Laser Scanning and Surface Analyses" at the University of Bonn has carried out various investigations regarding system calibration and long-term stability of sensors, for example in (Holst et al., 2018) and (Janßen et al., 2020). Effects of rain, fog and snow on LiDAR measurements have been investigated in (Rasshofer et al., 2011) for the case of automotive LiDAR. Further, (Muralikrishnan, 2021) discusses a study on performance evaluation of TLS and lists a summary of possible error sources. (Friedli et al., 2019) and (Schröder and Nowacki, 2021) both deal with refraction effects due to atmospheric conditions in terrestrial laser scanning. Further discussions regarding the influencing parameters on measurement data of a TLS can be found in (Gordon, 2008), (Kerekes and Schwieger, 2020), (Winiwarter et al., 2020) or (Wujanz et al., 2017).
The mentioned studies all deal with systematic errors on terrestrial laser scanning for incidental surveying or automotive LiDAR. With regard to multi-temporal deformation analysis, further aspects have to be considered. A significant influence comes from the referencing of the respective scans in a common coordinate system (Wujanz et al., 2018). One method for calculating transformation parameters uses artificial reference points that are distributed in the scanning area. An advantage of this method is that experience from conventional geodetic procedures can be adapted so that a familiar assessment of the results is possible. However, this methodology also has its disadvantages, for example (i) the complexity of installing the target points in the survey area, (ii) the required surveying of the targets to determine highly accurate reference coordinates and (iii) the, in practice often limited extent of the placed targets within the area of interest. In addition, due to the limited resolution capability of a scanner at long ranges $(>1 \mathrm{~km})$, the artificial markers have to be enlarged disproportionately, which hinders a useful application (Wujanz et al., 2018).

Another possibility is direct georeferencing (Paffenholz et al., 2010); (Reshetyuk, 2010). A third essential method for referencing different point clouds in a common reference frame is data-based and overcomes the disadvantage of considering the geometry of a measured object that is present based on a highresolution point cloud. (Wujanz et al., 2013) and (Friedli and Wieser, 2016) describe the methodology in detail and optimize the procedure with regard to deformation analysis. The entire error budget for a deformation analysis, taking all influences into account, is summarized in (Kauker and Schwieger, 2017) and (Harmening and Neuner, 2020).

\section{DATA AND METHOD}

Our experimental set up and data set allow us to investigate the specific consequences for the data quality of consecutive point clouds for environmental monitoring. We focus in our research on the last three of above mentioned error sources: atmospheric conditions and environment, object properties and scanning geometry as all of them contribute to the relative errors between consecutively acquired point clouds.

Due to the physical construction on a fixed metal frame in our experiment, it is hypothesized that the scanner will maintain its fixed position and the SOCS (Scanner's Own Coordinate System) of each individual scan will match. Thus, in the ideal case no further registration or alignment of the data set would be needed. However, environmental influences as well as our measurement activities can cause small changes to the scanning geometry or the georeferencing. Atmospheric conditions and environment include wind moving the laser scanner, rain obstructing the view and temperature causing displacements due to expansion of material used to fix the laser scanner. Object properties can be observed especially in the case of heavy rain. Changes in soil moisture on the beach influence reflective properties as for example reported by Jin et al. (Jin et al., 2021) and rain water collecting on paved surfaces prevents capturing them with laser scanning.

In the remainder of this section we introduce the data that was used for this study and present the methods.

\subsection{Data Description}

For this study we use data from a Riegl VZ-2000 laser scanner, which is operating continuously from the balcony of Grand 


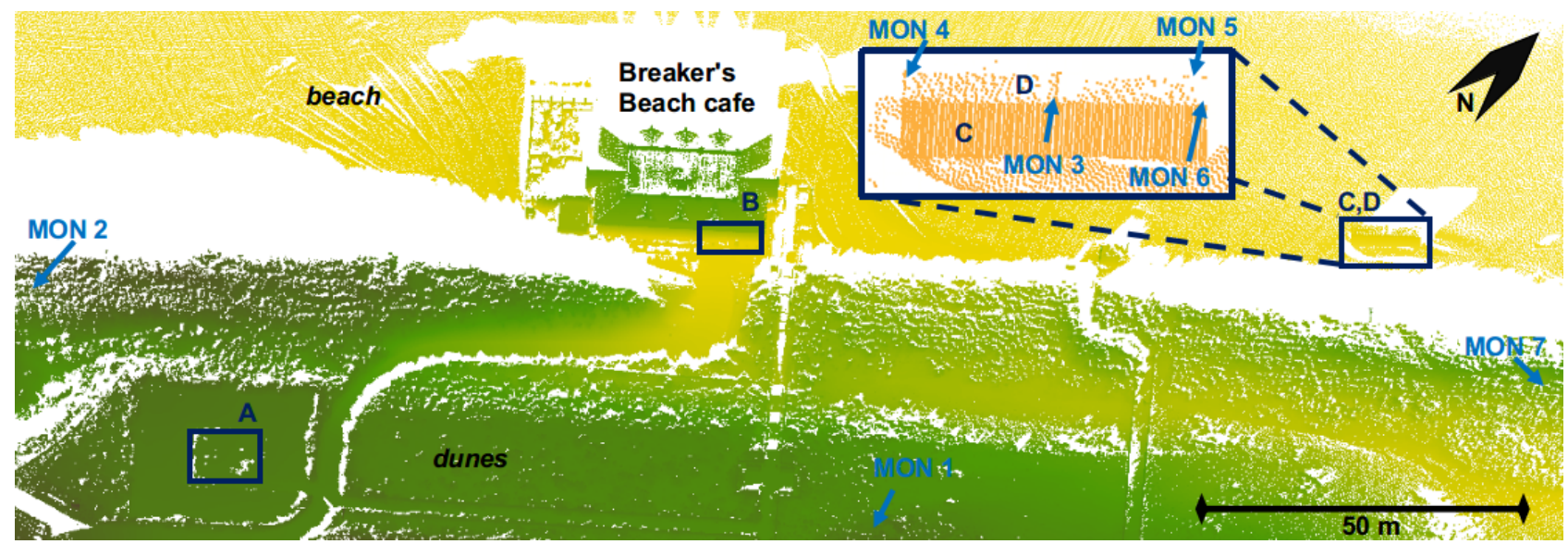

Figure 2. Point cloud of the area of interest from 20/02/2020, 01:00 with reference surfaces marked by letters A-D (compare Table 2) and location of GNSS sensors on the roof of the container and in the dunes (MON 1 - 7).

\begin{tabular}{|l|l|l|}
\hline Sensor & Description & Period \\
\hline Laser Scanner & Riegl VZ-2000 & $30 \mathrm{~min}$ \\
Weather station & Davis Vantage Pro 2 & $30 \mathrm{~min}$ \\
Inclination Sensors & Internal of Riegl & $1 \mathrm{sec}$ \\
& External of PC-IN1-1 & $15 \mathrm{sec}$ \\
GNSS sensors & DMT SAFEGUARD GNSS & $10 \mathrm{sec}$ \\
& Dutch Permanent GNSS & $30 \mathrm{sec}$ \\
\hline
\end{tabular}

Table 1. Specifications of all sensors used to collect data for this analysis.

Hotel Huis ter Duin in Noordwijk, The Netherlands since July 2019. A laser scan of the beach and dunes is taken every 30 minutes (see Figure 2). The measurement programme is selected with $100 \mathrm{kHz}$ and a spatial resolution of $30 \mathrm{mdeg}$. The beam divergence is $0.30 \mathrm{mrad}$. Assuming an orthogonal angle of incidence, this represents a point spacing of approx. $10 \mathrm{~cm}$ at a distance of $200 \mathrm{~m}$ and a footprint size of approximately $5 \mathrm{~cm}$. From the PLS data set, two subsets are chosen for the analysis in this paper: covering 8th to 11 th Feburary 2020 and 20th to 24th February 2020. In both time spans, two days have been relatively calm weather conditions and during one day there has been a heavy storm (i.e. storm 'Ciara' on 9/02/2020 and storm 'Yulia' on 22/02/2020). This selection was chosen to assess the influence of weather on the measurement results. For each of these time periods one scan every hour (taken at the beginning of every hour) was selected for further analysis.

Besides an extensive data set of point clouds, various other sensors were utilized during the scan experiment. A list of all sensors is summarized in Table 1. The analysed data includes recordings of

- inclination angles at $1 \mathrm{~Hz}$ from the scanner's (internal) sensors

- inclination angles from two external inclination sensors fixed to the base of the frame of the laser scanner (summarized to one value every 15 seconds)

- temperature, air pressure, humidity, wind speed, wind direction and precipitation data (every $30 \mathrm{~min}$ ) from a Davis weather station mounted next to the laser scanner

- Global navigation satellite system (GNSS) data from seven multi-sensor stations within scanned area

- GNSS data from the Dutch Permanent GNSS Array (Stations IJmuiden and Delft) as stable reference

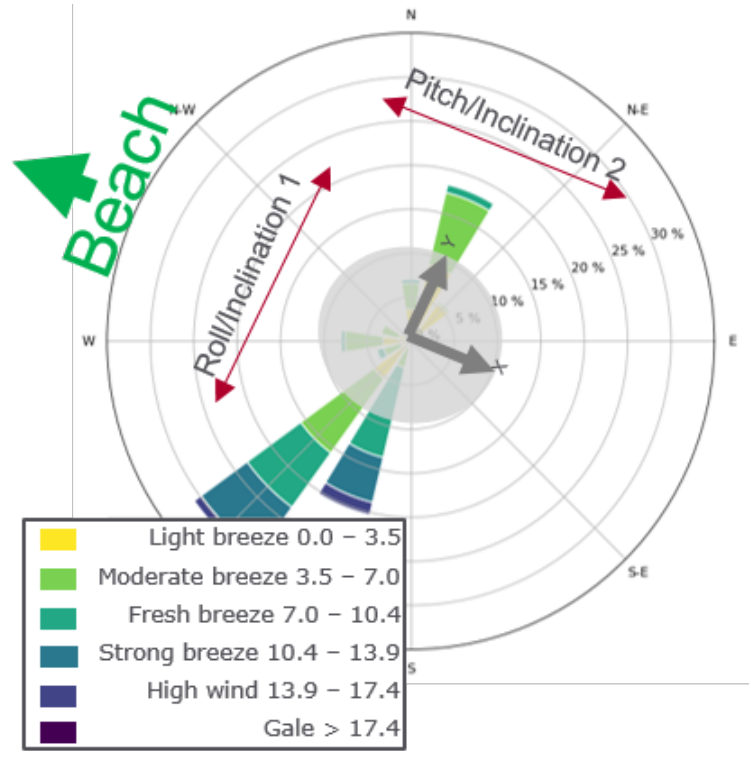

Figure 3. The relative location of the inclination sensors with respect to the laser scanner (at the center) and the beach (to the North-West) is indicated. In addition the main prevailing wind direction during the observation period is shown.

Measurement data from the internal inclination sensors are available during the entire measurement period. From $21 / 02 / 2020$ to $18 / 03 / 2020$, additional inclination readings are available from external sensors that are installed on the metal frame of the scanner mounting. In Figure 3 the pitch and roll directions of deviation from the center of the laser scanner are shown with respect to the beach and dominating wind direction from South-West.

\begin{tabular}{|l|c|c|c|}
\hline Surface & Size $\left[\mathrm{m}^{2}\right]$ & Points & Range [m] \\
\hline Helipad (A) & 124 & 9000 & 176.3 \\
Breaker's wall (B) & 5 & 720 & 162.5 \\
Container wall (C) & 28 & 480 & 206.1 \\
Container roof (D) & 27 & 2100 & 206.6 \\
\hline
\end{tabular}

Table 2. Reference surfaces with labels as in Figure 2 in brackets, average size, average number of points and average distance to the laser scanner (range). 


\subsection{Methods}

To detect the cause of the systematic effect, which among other things is responsible for the effect shown in Figure 1, our method consists of two parts: The first part on the influence of the scanning geometry deals with verifying the stability of the laser scanner's set up in Noordwijk. We are analysing the internal and external inclination sensors with regard to periodic and linear trends so that significant signals can be extracted. The second part is the analysis of the recorded data in the survey area. For this we inspect selected geometries and analyze the temporal variability in the period observed. Apparent deformations are compared with external sensors such as GNSS.

3.2.1 Stability of the laser scanner We installed inclination sensors on the metal frame of the laser scanner and utilized the laser's internal inclination sensors. By using the inclination sensors, two out of six degrees of freedom are measured and determined with regard to the positioning of the scanner. The three-dimensional translation as well as the orientation around the vertical axis are not measured externally.

In order to be able to describe the behaviour of the scanner position in relation to time, all data from the internal and external inclination sensors between 8 February and 18 March are initially used for an analysis. The external sensors, with an accuracy of $0.0175 \mathrm{~mm} / \mathrm{m}$, are more sensitive to movements than the internal sensors with $0.14 \mathrm{~mm} / \mathrm{m}$. Each data set (Inclination 1, Inclination 2, Roll and Pitch) is initially inspected with respect to dominant periodic components by means of a discrete Fourier transformation. The discrete-time signal is transferred into a frequency spectrum and the diurnal dependence of the inclination values is verified. The confirmation of the diurnal cycle serves as an input variable for a time series decomposition. The time series is decomposed into its core components and considered as the sum of trend, period and residuals. This procedure provides conclusions about low- and high-frequency components within the time series. Low frequency in this context implies that a period lasts at least as long as a single scan. The scan is affected as a whole but individual data within the scan is not affected relative to other data within the same scan. High frequency here refers to a period that is below the scan duration. Individual scan lines are thus adversely affected.

The data from the inclination sensors are divided into two parameters using this decomposition. The data is aggregated into 30-minute segments. The result is a mean inclination, which represents the trend or periodicity, and a standard deviation, which describes the measurement noise of the inclination sensors. Next to the laser scanner a Davis weather station (Davis vantage Pro 2) was mounted to record temperature, precipitation and wind speed and direction every 30 minutes. The respective parameters are subsequently analysed with regard to a correlation with weather data.

3.2.2 Influence of Scanning Geometry The next step is to detect in the point cloud data the effects that are identified at the scanner's position. Within the field of view of the laser scanner, we chose three reference objects, as summarized in Table 2: paved helipad, with a slight slope of about $1.4 \mathrm{~cm}$ per meter and dimensions of $10.6 \mathrm{~m} \times 11.7 \mathrm{~m}$ at $176 \mathrm{~m}$ range, the wall of the Breakers's Beach cafe, right in front of the laser scanner at about $163 \mathrm{~m}$ range and about $5 \mathrm{~m}^{2}$ in size, and two containers placed on the beach at about $206 \mathrm{~m}$ distance from the laser scanner. We analysed the data by fitting planes through the stable reference planes using principal component analysis (PCA). We then extract the mean $\mathrm{z}$-coordinate of the helipad, the mean $\mathrm{x}$ coordinate of the wall of the cafe and the mean coordinates as well as normal vectors of the containers and the residuals with respect to these planes. The root mean squared sum (RMSS) of the residuals of the plane analyses are indicators of lower quality scans.

We establish a correlation with the inclination values on the scanner and the weather data to derive a potential cause. The mean coordinates give an indication of the relative position of the reference geometry with respect to the reference frame in which the scanning data is coordinated. Variability give indications of long term stability and orientation of the laser scanner. These in turn are compared with the orientation from the internal inclination sensor, verified by the externally mounted inclination sensors. During the measurement campaign, seven GNSS receivers were installed in the area of the dunes and on the shipping containers on the beach (labelled MON 1 - MON 7, see Figure 2). The GNSS baseline processor Wa2 (Wanninger, 2020) is used for the evaluations. Movement as well as stability of the container is checked against GNSS measurements. The GNSS measurements are processed strictly by means of baselines. The absolute coordination is done via two reference stations of the Dutch Permanent GNSS Array (DPGA) in IJmuiden and Delft. Local reference stations, whose position is stable during our experiment, coordinates the object points on the container. We obtain $3 \mathrm{D}$ coordinates with an accuracy of a few millimetres.

\section{RESULTS}

The results are presented in four parts: First, we evaluate the inclination sensors on the scanner and then correlate the measured values with the weather data. Subsequently we relate our results to effects in point cloud data and verify them with independent GNSS measurements.

\subsection{Inclination Sensors}

First, the stability of the scanner position is checked by means of the external and internal inclination sensors. The maximum deviations in the data are up to $+/-20 \mathrm{~mm} / \mathrm{m}$ in the majority of the time (occasionally up to $+/-80 \mathrm{~mm} / \mathrm{m}$ ) for the internal sensors and $+/-0.1 \mathrm{~mm} / \mathrm{m}$ for the external sensors. This indicates that the scanner generally has a stable foundation in connection with the hotel. In relation to the maximum values, no significant movement is detectable, which is measured at the base of the fixture. Pessimistically, the value of $+/-0.1$ $\mathrm{mm} / \mathrm{m}$ corresponds to a deviation of $20 \mathrm{~mm}$ in the measured $\mathrm{z}$-coordinate at $200 \mathrm{~m}$ distance. This is within the measurement accuracy as well as the spatial resolution of the scanner. With a discrete Fourier transform on the raw data of the external sensor system a dominant signal is registered in the frequency spectrum with a period of 24.5 hours. A subsequent extraction of the core components of the signal in trend, period and noise yields the results summarized in Table 3 .

Trend and periodicity have a low frequency nature and do not affect the measurement data within a scan. The effect of trend and period on the point cloud data can be corrected in the form of a six parameter transformation. The period, which is detected with the external sensors, is not present in the data from the internal sensors. The measurement noise of the sensors overlays the periodic signal. The remaining signal include the inaccuracies of the measurement systems, but also signals that 


\begin{tabular}{|c|c|c|c|c|c|c|c|c|}
\hline & \multicolumn{3}{|c|}{ Trend } & \multicolumn{3}{c|}{ Period } & \multicolumn{3}{c|}{ Residual Signal } \\
\hline & Min & Max & Avg & Min & Max & Min & Max & Avg \\
\hline Incl 1 & -0.058 & 0.054 & 0.002 & -0.018 & 0.016 & -0.108 & 0.104 & 0.016 \\
Incl 2 & -0.110 & 0.080 & 0.000 & -0.023 & 0.017 & -0.185 & 0.226 & 0.033 \\
Roll & -0.223 & 0.112 & -0.008 & - & - & -33.185 & 28.666 & 1.016 \\
Pitch & -0.151 & 0.346 & -0.007 & - & - & -55.670 & 71.644 & 1.645 \\
\hline
\end{tabular}

Table 3. Trend, period and residual signal of the respective inclination signals. Incl 1 and Incl 2 refer to the external inclination sensors. All values in $\mathrm{mm} / \mathrm{m}$. The highest values are marked in blue.

cannot be modelled by means of trend and period. This is an indicator for high-frequency movements of the scanner and which therefore influences the data within a scan. It should be noted that increased values visually coincide with storm events, as can be seen in Figure 4. The deviations in the external sensors deviate only insignificantly from the measurement accuracy of the inclination measurement, except for a few occasions which coincide with strong storm events. The values indicate that the scanner is sufficiently stabilized and anchored. The remaining signal of the internal sensors show a higher noise (cf. Table 3 , marked in blue), which cannot be explained by the measurement accuracy alone. Visually, the values correlate with increasing wind speed (Figure 4), which leads to the following step in our analysis.

\subsection{Correlation to Weather Data}

For this part of the analysis we focus on the two selected four day periods. First, we aggregate the inclination data into blocks of 30 minutes and calculate an average value for each block. Averaging minimizes high-frequency noise and this value is to be correlated with the temperature and wind data as a parameter for periodic and trend-related behaviour. In addition to the average value, a standard deviation of the respective inclination values is calculated for each block. The standard deviation is an indicator for the statistical distribution of the individual measured values and therefore describes the movement of the sensor within a block, which allows to detect the highfrequency component. The values are processed graphically and correlation analyses are conducted to investigate the relation with the weather conditions. Figure 4 shows the pitch and roll mean values with their standard deviations during both storms, together with wind speed and temperature data. Visually large standard deviations in the inclination values coincide with the peaks of the wind speed during both storms.

\begin{tabular}{|l|l|c|c|}
\hline \multicolumn{2}{|l|}{} & Temperature & Wind Speed \\
\hline \multirow{2}{*}{ Roll } & Mean & 0.03 & 0.05 \\
& Std. & 0.17 & 0.51 \\
\hline \multirow{2}{*}{ Pitch } & Mean & 0.12 & -0.01 \\
& Std. & 0.23 & 0.71 \\
\hline \multirow{2}{*}{ Incl 1 } & Mean & 0.80 & 0.33 \\
& Std. & 0.25 & 0.72 \\
\hline \multirow{2}{*}{ Incl 2 } & Mean & -0.41 & -0.19 \\
& Std. & 0.25 & 0.61 \\
\hline
\end{tabular}

Table 4. Pearson correlation coefficients of mean values and standard deviations of pitch and roll with temperature and wind speed respectively. The significant correlation values are marked in blue.

The correlation analysis (cf. Table 4) confirms our assumption that the wind and storm events are the cause of the deviations in the scans. The Pearson correlation coefficient was calculated for the mean values and standard deviations of inclination values from both sensors with temperature and wind speed respectively. Wind speed correlates with the standard deviation of the inclination values between 0.51 (roll, internal sensor) and
0.72 (inclination 1, external sensor) and the mean of inclination 1 correlates 0.8 with temperature. It is important to note that these are relative values and no absolute magnitudes of changes can be derived from level of correlation. In the high-frequency range, we see a high correlation with wind speed for all inclination sensors. From this we can conclude that the storm events are the cause of the systematic deviations in the scans described. The external inclination sensor is sensitive enough to detect deviations in the scanners position caused by temperature changes. The dominating 24.5 day frequency indicates a temperature-dependent diurnal variation. To verify these findings and relate them to the effects on the point cloud data, the influences in the point cloud data is discussed in the following subsection.

\subsection{Point Cloud Data Quality}

The analysis of the scans is done by fitting planes to parts of the point cloud sampling planar patches (as listed in Table 2). For the comparison, an average main orientation (e.g. in the case of the helipad in the z-direction) and the RMSS of the residuals are calculated. The mean value of the $\mathrm{z}$-coordinate (for the helipad, $\mathrm{x}$-coordinate for vertical planes) is an indicator for the lowfrequency component and the residuals for the high-frequency components. Larger shifts, as well as small variations in the position of the laser scanner naturally appear in the point clouds as well. In the first measurement period, a larger jump in the deviation (in the order of $5 \mathrm{~cm}$ ) correlates with the beginning of storm Ciara on 09/02/2020. The second caused by our own maintenance activity on 20/02/2020 around 12:00, resulted in an apparent shift of the helipad of about $4 \mathrm{~cm}$ in z-direction (marked with arrow in Figure 5) and the wall of the Breaker's Beach cafe shifted by about $2 \mathrm{~cm}$ in $\mathrm{x}$-direction. These movements can be compensated by deriving transformation matrices, that describe the rigid transformation of the point cloud. Such a transformation is described by means of six parameters. A translation in $\mathrm{x}-, \mathrm{y}-$ and $\mathrm{z}$-direction and a rotation around the corresponding axes. For this estimation, the iterative-closestpoint (ICP) algorithm as implemented in RiSCAN PRO (V2.11) is used (Gaisecker et al., 2012).

After applying the rigid transformations, the remaining high frequency movement of the scanner is further analyzed and compared with data from the weather station. During calm weather conditions the inclination sensors show standard deviations of less than $1 \mathrm{~mm} / \mathrm{m}$ in both pitch and roll. For wind speeds around $10 \mathrm{~m} / \mathrm{s}$ and higher the standard deviations of pitch and roll are reaching above $5 \mathrm{~mm} / \mathrm{m}$ and above $3 \mathrm{~mm} / \mathrm{m}$ respectively. These indicate that the scanner is shaking while scanning. The effect of this is found in the point clouds as radial stripes, coming from the laser scanner, visible mostly on flat surfaces (see Figure 1). To quantify this effect, we compared the residuals from a fitted plane through the references surfaces on the helipad and the wall of the beach cafe, shown in Figure 5. The sum of residuals on these two surfaces rises up to $14 \mathrm{~mm} / \mathrm{m}$ at the peak of the storm. Additionally we observe 

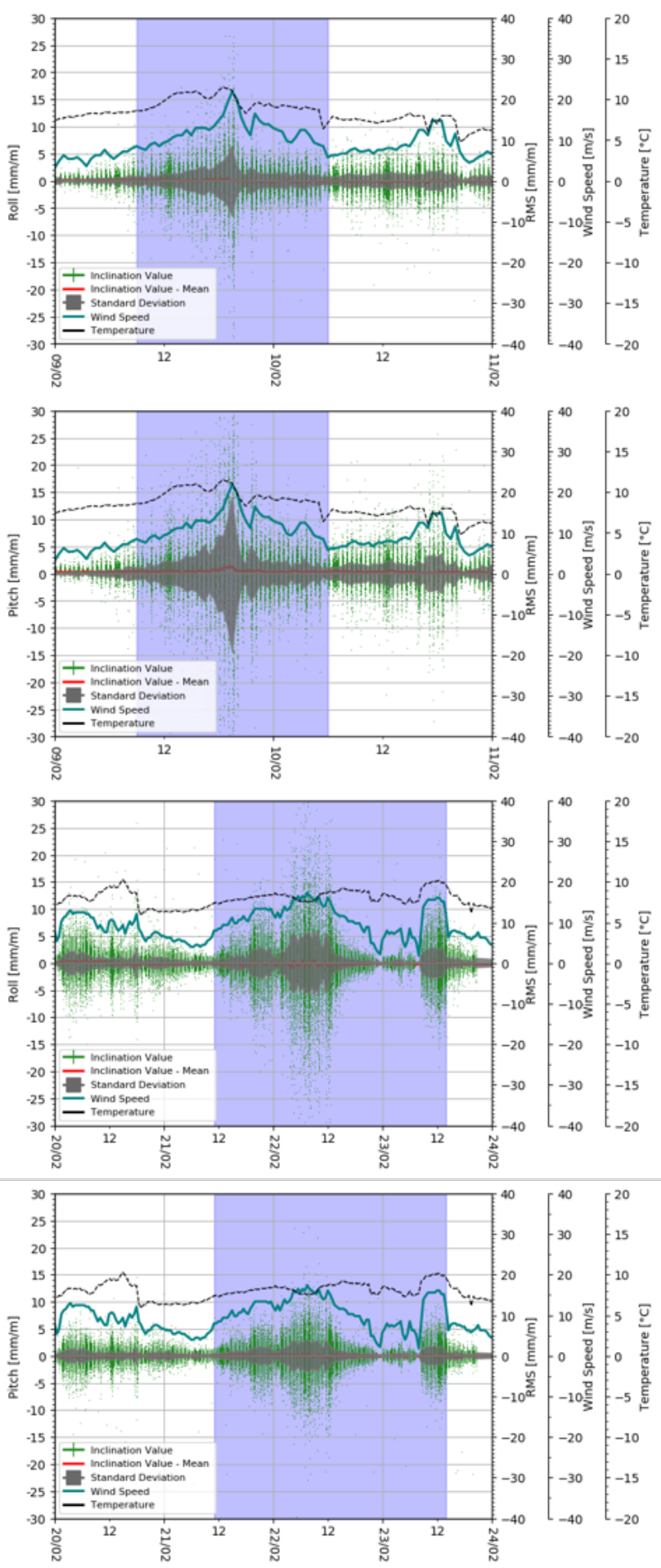

Figure 4. Internal inclination values during observation periods around storm Ciara (two top panels) and storm Yulia (two bottom panels). The stormy periods are marked with a blue background. Raw data, mean values and standard deviations of the inclination data are compared to temperature and wind speed.
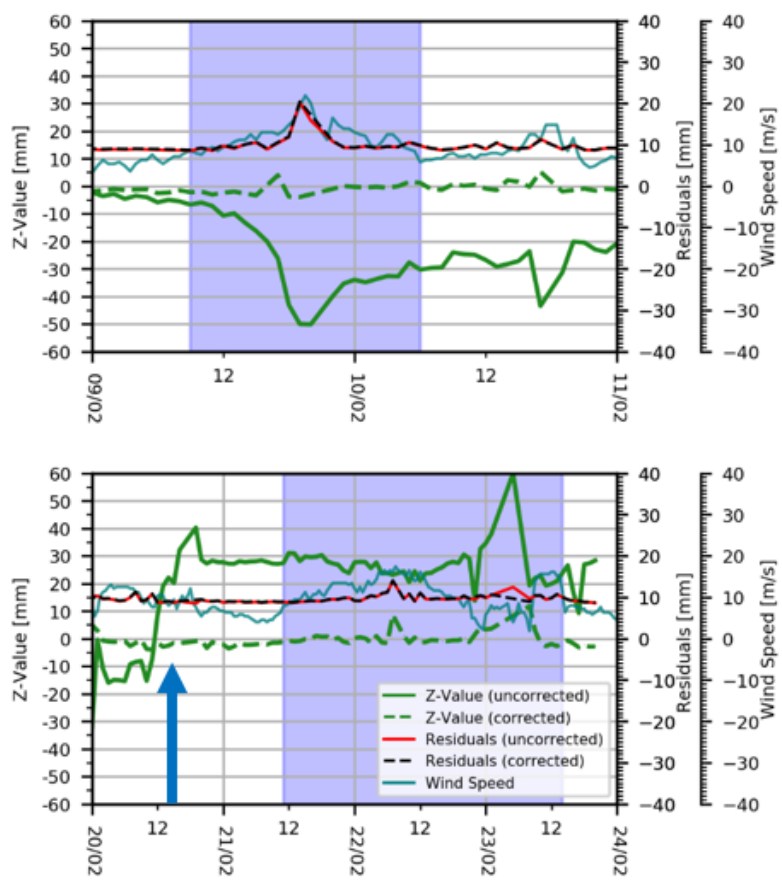

Figure 5. Plane estimation at helipad and comparison of the parameters with the weather data during storm Ciara (top panel) and storm Yulia (bottom panel). The stormy periods are marked with a blue background. The jump (marked with arrow) in the mean z-coordinate of the helipad around noon on 20/02/2020 was caused by maintenance activities.

that heavy rain does not allow data collection on the helipad, as the wet paved ground does not return any signal. The residuals on the wall of the Breaker's Beach cafe reach up to double the values in calm conditions (e.g. no rain and wind below 10 $\mathrm{m} / \mathrm{s}$ ) during heavy rain, owing to water droplets in the air, on the glass of the laser scanner itself and dripping from the roof directly in front of the wall.

\subsection{Combination with Data from GNSS Sensors}

To investigate the stability of the laser scanner by an independent measurement, single frequency GNSS receivers were used. Three reference stations in the dunes were evaluated together with two reference stations of the Dutch Permanent GNSS Array (DPGA) in IJmuiden and Delft. GNSS raw data are recorded in this network with a frequency of 30 seconds and combined to coordinate solutions of $1,3,6,12$ and 24 hours. No significant movement can be detected in the observation period. The measurements are calculated with a 3D accuracy of 2.6 $\mathrm{cm}$. Due to the demonstrated stability, these stations can in turn be used as base stations for the GNSS calculations on the container. Due to the short baselines to the stations in the dunes, the coordinates on the container are calculated with an accuracy of $5 \mathrm{~mm}$. Planes are estimated from the four GNSS coordinates and the selected points of the scan data. The parameters of the plane estimation are used as a benchmark for both measurement systems. The laser scans are not referenced in a global coordinate system, so an absolute comparison with the GNSS data is not possible. However, a relative comparison of the plane change is available. In Figure 6, the first GNSS measurement on 21 February is taken as a reference and change in vertical angle is considered. In the data of the laser scanner, a higher measurement noise can be seen, which indicates the reduced 


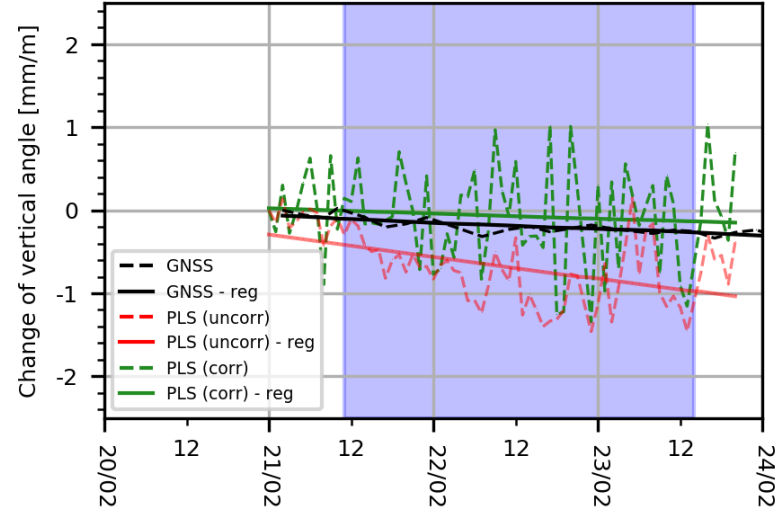

Figure 6. Angular change between container and horizontal: Comparison of the plane modelling from uncorrected (uncorr), corrected (corr) point cloud data (PLS) and the four GNSS antennas. The stormy periods are marked in blue.

measurement accuracy. For the comparison, a linear regression (reg) is performed for each data set. The downward trend shows that the container inclines towards point MON 4 during the two storm days. The offset between the graphs indicates that the coordinate systems are not identical. The z-coordinate of the PLS is not perpendicular because the scanner is not horizontal. Uncorrected data show a stronger slope, which is caused by the low-frequency rigid movement. After rigid body transformation, the offset remains (due to different coordinate systems) but the relative change is identical between PLS and GNSS.

\section{DISCUSSION}

Most systematic influences (e.g. displacement of the scanner position, propagation of the laser or interaction with the object to be measured) are time-dependent, and have a diurnal variation or depend on temperature. Thus, correlation calculations between the individual measured variables must be made with some caution. A high correlation doesn't necessarily imply the existence of a causal relationship, but in combination with several data sources and methods these relationships can be established with high probability.

The analysis of the data from inclination sensors on the frame of the scanner (external) and in the laser scanner (internal) show that a decline in data quality is observed when the standard deviation of the inclination angles during a scan is higher than 4 $\mathrm{mm} / \mathrm{m}$. These events lead to high (in the order of several centimeters) sum of residuals on flat, stable reference surfaces and thus an increase in noise in the point cloud overall. The effect, that we quantify as sum of residuals on flat reference plans is assumed to be present in the entire point cloud, but is less visible on rough and more dynamic surfaces. It does render elevation values less accurate. Temperature related diurnal periodic signals in inclination of the laser scanner can be observed with the external inclination sensors. However, they do not show significant effects on the point clouds in our data set.

These effects of observed high frequency movement related to point cloud data quality are in our case linked to extreme weather events (or maintenance activities). If the laser scanner is subject to strong winds with wind speed of $10 \mathrm{~m} / \mathrm{s}$ and higher, the standard deviations of the inclination angles rise and the data quality declines. Our experimental set up including a weather station directly next to the laser scanner allows to link these events and high correlation leads us to the conclusion of a very probable causal relationship. Heavy rain $(>4 \mathrm{~mm} /$ hour $)$ effects data quality as well, by introducing noise through reflections from rain drops, as well as gaps in the data due to accumulated water and wet surfaces.

Rigid movement of the laser scanner, detected with the external and internal inclination sensors, leads to misalignment of consecutive point clouds. These misalignment can be corrected with rigid body transformations, derived from the data itself or from the measured inclination angles. Methods to estimate this transformation can be sensor-based, by means of artificial targets or data-based with the use of an ICP algorithm (Wujanz et al., 2018). The radial stripes, that lead to high sum of residuals on reference planes as effect of high frequency movement of the scanner are much more challenging to correct for.

The analysis of the GNSS sensors on a reference object as the shipping containers show that to a certain extend GNSS data can be used as validation data. The accuracy of the GNSS measurements in the order of $\mathrm{mm}$ allows for the verification of general trends, such as stability of a set up or gradual movement of a (reference) object into one direction. Where possible, it is recommended to add an additional measurement technique, such as GNSS, which provides independent results.

\section{CONCLUSIONS}

External influences significantly affect the data quality of multitemporal laser scanner data. We have shown that weather, especially storm events, have a non-negligible influence. A weather station in the close vicinity of the laser scanner helps to identify conditions with critical effects on the data. The internal inclination sensors of the Riegl VZ-2000 allow to indicate if a scan is significantly shifted and/or contains higher noise levels. Strong gusts of wind can shift the scanner, causing deviations of around $5 \mathrm{~cm}$ at $175 \mathrm{~m}$ range, and wind speeds of $10 \mathrm{~m} / \mathrm{s}$ and higher cause shaking of the scanner, which leads to noisy representations of flat surfaces. For the direct correction of the data, the movements must be decomposed into high and low frequency components, whereby the decision threshold is the measurement duration of a single scan. Low-frequency components can be taken into account by a correction by a 6-parameter rigid body transformation. In the high-frequency range, a correction via high-frequency measuring inclination sensors is conceivable, but time synchronisation of the data sets is the challenge here.

\section{ACKNOWLEDGEMENTS}

The authors would like to thank Sander Vos for his major contribution to the PLS data acquisition and maintenance of the permanently mounted laser scanner as well as the weather station during the entire field work campaign. He also organized the shipping containers placed on the beach. Thanks go also to Grand Hotel Huis ter Duin for their cooperation and to DMT for the set-up of reflectors and GNSS receivers. This research has been supported by the Netherlands Organization for Scientific Research (NWO, grant no. 16352) as part of the Open Technology Programme, by Rijkswaterstaat (Dutch Ministry of Infrastructure and Water Management) and by the European Coal and Steel Research Fund [RFCS project number 800689 (2018)]. 


\section{REFERENCES}

Anders, K., Lindenbergh, R. C., Vos, S. E., Mara, H., de Vries, S., Höfle, B., 2019. High-frequency 3D geomorphic observation using hourly terrestrial laser scanning data of a sandy beach. ISPRS Annals of Photogrammetry, Remote Sensing and Spatial Information Sciences, IV-2-W5, 317-324.

Friedli, E., Presl, R., Wieser, A., 2019. Influence of atmospheric refraction on terrestrial laser scanning at long range. Proceedings of the 4th Joint International Symposium on Deformation Monitoring (JISDM 2019), 15-17 May 2019, JISDM, Athens, Greece.

Friedli, E., Wieser, A., 2016. Identification of stable surfaces within point clouds for areal deformation monitoring. Proceedings of the 3rd Joint International Symposium on Deformation Monitoring (JISDM 2016), 30 March - 1 April 2016, Vienna, Austria.

Gaisecker, T., Pfennigbauer, M., Sevcik, C., Studnicka, N., 2012. Terrestrisches Laser Scanning in den Alpen mit dem RIEGL VZ-4000 - für Geländeerfassung, Hangrutschungsüberwachung und Gletschermonitoring. Österreichische Zeitschrift für Vermessung und Geoinformation, 1, 45-52.

Gordon, B., 2008. Zur Bestimmung von Messunsicherheiten terrestrischer Laserscanner. PhD thesis, TU Darmstadt.

Harmening, C., Neuner, H., 2020. A spatio-temporal deformation model for laser scanning point clouds. Journal of Geodesy, 94(2), 1-25.

Holst, C., Medic, T., Kuhlmann, H., 2018. Journal of Applied Geodesy, 12(2), 169-185.

Janßen, J., Kuhlmann, H., Holst, C., 2020. Assessing the temporal stability of terrestrial laser scanners during long-term measurements. Contributions to International Conferences on Engineering Surveying, Springer, 69-84.

Jin, J., Verbeurgt, J., De Sloover, L., Stal, C., Deruyter, G., Montreuil, A.-L., Vos, S., De Maeyer, P., De Wulf, A., 2021. Monitoring spatiotemporal variation in beach surface moisture using a long-range terrestrial laser scanner. ISPRS Journal of Photogrammetry and Remote Sensing, 173, 195-208.

Kauker, S., Schwieger, V., 2017. A synthetic covariance matrix for monitoring by terrestrial laser scanning. Journal of Applied Geodesy, 11(2), 77-87.

Kerekes, G., Schwieger, V., 2020. Elementary Error Model Applied to Terrestrial Laser Scanning Measurements: Study Case Arch Dam Kops. Mathematics, 8, 593.

Kuschnerus, M., Lindenbergh, R., Vos, S., 2021. Coastal change patterns from time series clustering of permanent laser scan data. Earth Surface Dynamics, 9(1), 89-103.

Lichti, D. D., 2010. Terrestrial laser scanner self-calibration: Correlation sources and their mitigation. ISPRS Journal of Photogrammetry and Remote Sensing, 65(1), 93-102.

Muralikrishnan, B., 2021. Performance evaluation of terrestrial laser scanners - a review. Measurement Science and Technology. http://iopscience.iop.org/article/10.1088/13616501/abdae3. preprint.
Paffenholz, J.-A., Alkhatib, H., Kutterer, H., 2010. Direct georeferencing of a static terrestrial laser scanner. Journal of Applied Geodesy, 4, 115-126.

Rasshofer, R. H., Spies, M., Spies, H., 2011. Influences of weather phenomena on automotive laser radar systems. Advances in Radio Science, 9, 49-60.

Reshetyuk, Y., 2010. Direct Georeferencing with GPS in Terrestrial Laser Scanning. ZFV - Zeitschrift fur Geodasie, Geoinformation und Landmanagement, 135, 151-159.

Schröder, D., Klonowski, J., 2019. i²mon - integrated monitoring for the detection of ground and surface displacements caused by coal mining. Proceedings of the 4th Joint International Symposium on Deformation Monitoring (JISDM 2019), 15-17 May 2019, Athens, Greece.

Schröder, D., Nowacki, A., 2021. Die atmosphäre als restriktiver einfluss auf messergebnisse eines long range laserscanners. Proceedings of 21. Internationale Geodätische Woche 2021, Obergurgl, Austria.

Soudarissanane, S., Lindenbergh, R., Menenti, M., Teunissen, P., 2011. Scanning geometry: Influencing factor on the quality of terrestrial laser scanning points. ISPRS journal of photogrammetry and remote sensing, 66(4), 389-399.

Vos, S., Kuschnerus, M., de Vries, S., Lindenbergh, R., 2020a. Coastscan: Data of daily scans at low tide Kijkduin January 2017. https://doi.org/10.4121/uuid:409d3634-0f52-49ea-8047aeb0fefe 78af. Dataset.

Vos, S., Kuschnerus, M., Lindenbergh, R. C., 2020b. Assessing the error budget for permanent laser scanning on coastal beaches. Proceedings of FIG Working Week 2020, Amsterdam, The Netherlands.

Vos, S., Lindenbergh, R., de Vries, S., 2017. CoastScan: Continuous monitoring of coastal change using terrestrial laser scanning. Proceedings of Coastal Dynamics 2017, Helsingør, Denmark, 1518-1528.

Wanninger, 2020. Gnss baseline processing engine wa2. http://www.wasoft.de/e/wa2/index.html. Software.

Winiwarter, L., Anders, K., Höfle, B., 2020. Herausforderungen in der fehlerfortpflanzung von laserscandaten für multitemporale analysen zur verbesserten quantifizierung des level of detection. 40. Wissenschaftlich-Technische Jahrestagung der DGPF - Publikationen der DGPF, Band 29.

Wujanz, D., Avian, M., Krueger, D., Neitzel, F., 2018. Identification of stable areas in unreferenced laser scans for automated geomorphometric monitoring. Earth Surface Dynamics, 6(2), 303-317.

Wujanz, D., Burger, M., Mettenleiter, M., Neitzel, F., 2017. An intensity-based stochastic model for terrestrial laser scanners. ISPRS Journal of Photogrammetry and Remote Sensing, 125, 146-155.

Wujanz, D., Krueger, D., Neitzel, F., 2013. Defo scan++: Surface based registration of terrestrial laser scans for deformation monitoring. Proceedings of the 2nd Joint International Symposium on Deformation Monitoring (JISDM 2013), 09-10 September 2013, 7 .

Wunderlich, T., Niemeier, W., Wujanz, D., Holst, C., Neitzel, F., Kuhlmann, H., 2016. Areal deformation analysis from TLS point clouds-The challenge. Allg. Vermess., 123, 340-351. 